\title{
Three-dimensional tracking of the long time trajectories of suspended particles in a lid-driven cavity flow
}

Received: 18 April 2005/ Revised: 1 October 2005/ Accepted: 8 October 2005 / Published online: 11 November 2005

(C) Springer-Verlag 2005

\begin{abstract}
Stereo imaging methods are used to measure the positions of solid spherical particles suspended in a viscous liquid and enclosed in a transparent cubic cavity. The liquid and particle motions are driven at the top lid by a conveyor belt operated at constant speed. Based on sequences of stereo views of the full cavity, the particles are tracked continuously along their three-dimensional orbits. The corresponding position histories are treated as noisy stochastic data and processed using Kalman filters to fill data gaps and attenuate the effect of measurement errors. The lid-driven viscous flow is characterised by an intricate internal structure which is mirrored in the particle paths. The tracks of the solid particles align with long exposure images of laser-illuminated micro-particles in selected transverse planes. Nevertheless, their long time trajectories appear to cluster along preferential pathways of the internal circulation pattern.
\end{abstract}

\section{Introduction}

Fluid motions are often made visible by seeding the flow with tracer particles. Much information can then be gained by registering particle displacements over short time intervals using particle imaging velocimetry (PIV) or particle tracking velocimetry (PTV). A single camera can be used to capture two-dimensional (2D) displacements, or stereo cameras can be used when 3D displacements are of interest. For fluids seeded with micro-particles, measurements are commonly facilitated by illuminating

S. J. Tsorng $\cdot$ H. Capart $(\bowtie) \cdot$ D. L. Young

Department of Civil Engineering, National Taiwan University, Taipei, 106 Taiwan, ROC

E-mail: hcapart@yahoo.com

J. S. Lai · S. J. Tsorng · H. Capart · D. L. Young Hydrotech Research Institute, National Taiwan University, Taipei, 106 Taiwan, ROC only a thin slice of a flow using a continuous or a pulsed laser. If the seeding is not too sparse, and if multiple slices can be acquired, collections of short particle tracks can then be assembled into detailed Eulerian flow fields (Adrian 1991, 2005).

An alternative is to adopt a Lagrangian point of view. Instead of short time displacements, one seeks to capture the long-term trajectories of the particles as they move about the domain. Valuable insights into the flow structure can then be gained from these long time orbits. For 3D flow fields, however, this approach runs into a number of difficulties. First, long trajectories cannot be registered using laser illumination because particles would move in and out of the light sheet. Next, the micro-particles which respond only to laser illumination are no longer appropriate, and one must work instead with visible solid particles of greater size. This in turn raises concerns about whether the motions of the solid particles do indeed closely reflect the motions of the embedding fluid. These concerns can be minimised by using sparsely seeded, neutrally buoyant particles. Yet even under such precautions, deviations may occur because of finite size effects or inertial migration (Han et al. 1999; Matas et al. 2004).

An intriguing example of cross-stream particle motions is provided by inertial migration in non-homogeneous laminar shear flows. The simplest case is that of Poiseuille flow, sparsely seeded with neutrally buoyant solid particles of finite size. Experimental observations show that the particles do not distribute themselves evenly over the cross-section of the circular pipe, instead they migrate towards ring-shaped regions of preferential concentration (Segré and Silberberg 1962; Matas et al. 2004). It is currently unclear how such effects play out in flows exhibiting a fully 3D internal structure.

Acquiring 3D particle trajectories over long time intervals further requires special imaging methods. Since a laser sheet cannot be used to sample particle positions within a known plane, full volume stereo imaging must be used. To reconstruct the trajectories of individual particles, furthermore, PTV must be adopted. Special 
procedures are also needed to attenuate measurement errors and fill gaps due to missed particles.

In the present work, we address these general issues in the context of a particular case. The flow configuration chosen is the lid-driven cubic cavity. This is a closed system, with a well-defined geometry and boundary motion. Liquid is enclosed between five rigid walls and driven by a top lid moving at constant speed. Despite its simplicity, the configuration generates complex flows, featuring $3 \mathrm{D}$ circulation and a rich vortex structure. This flow structure is now well documented thanks to a number of computational and experimental studies. An overall review of the fluid mechanics of driven cavities is provided by Shankar and Deshpande (2000). In three dimensions, computational solutions have been obtained both for Stokes flow (Murugesan et al. 2005), corresponding to vanishing Reynolds numbers, and for the full Navier-Stokes equations at Reynolds numbers up to $R e=2,000$ (Chiang et al. 1997, 1998; Sheu and Tsai 2002; Lo et al. 2005). In the laboratory, both dyes and passive tracer particles have been used to visualise and measure the flow field inside lid-driven cavities (Pan and Acrivos 1967; Koseff and Street 1984; Guermond et al. 2002; Migeon 2002). In most of these experiments, laser light sheets were used to highlight motions within 2D slices of the 3D flow.

To capture long time particle trajectories within the 3D cavity, the present work will rely on two main tools: PTV and Kalman filtering. PTV is now a well-established technique of experimental fluid mechanics (for review, see Adrian 1991). While not yet as mature as their 2D counterparts, 3D PTV techniques have been documented in a number of recent works (Maas et al. 1993; Ushijima and Tanaka 1996; Virant and Dracos 1997; Kieft et al. 2002). The stereoscopic PTV technique implemented in the present work builds on earlier works by Spinewine et al. (2003) and Douxchamps et al. (2005). Kalman filtering, on the other hand, is a signalprocessing technique widely used to deal with time series of noisy data. Introduced by Kalman (Kalman 1960; Kalman and Bucy 1961), the technique has been used in fluid mechanics for data assimilation (Ide and Ghil 1997) and flow control (Högberg et al. 2003; Lauga and Bewley 2004). In the present work, we apply this tool to the 3D particle trajectories in order to fill data gaps and attenuate measurement errors. For 2D PTV, Kalman filter was used for a similar purpose by $\mathrm{Hu}$ (2003). Beyond fluid mechanics, the target tracking problem has been the focus of much applied work in signal treatment (Shah et al. 1993; Castellari et al. 2001; Ito et al. 2001; Liao 2002).

The present paper is structured as follows. In the following section, the laboratory setup used to conduct the lid-driven cavity flow experiments is first presented. The methods used to position particles in the $2 \mathrm{D}$ image plane and inside the 3D viewing volume are then documented, along with corresponding error estimates. Next, the Kalman filter approach used to reduce the effect of positioning errors on the particle trajectories is detailed. Measurements of the trajectories of suspended particles in the lid-driven cavity are then described and compared with long exposure images of laser-illuminated micro-particles. Finally, some overall conclusions are proposed.

\section{Experimental setup}

Experiments are performed using the apparatus shown in Fig. 1. The phenomena of interest occur in a small cubic cavity having side $S=10 \mathrm{~cm}$, filled with liquid, in which a small number of neutrally buoyant solid spheres are suspended. This inner cavity has transparent rigid walls on five sides and a moving lid on the top side. The moving lid is obtained by placing on the open top face of the cavity an upside down conveyor belt system, driven by a variable speed motor. The inner cavity is itself placed inside a larger plane-walled enclosure. This outer tank is filled with liquid up to a level slightly above the conveyor belt lid. In this way, the lubrication flow that inevitably occurs through the small gaps between the conveyor belt and the top of the cavity side walls does not lead to liquid loss from the cavity.

The plastic particles used for the experiments have a diameter of $3 \mathrm{~mm}$ and a specific gravity $\mathrm{SG}=1.21$. As in the experiments of Matas et al. (2004), the liquid chosen is a mixture of glycerol and water having a density matched to the density of the particles. Neutral buoyancy is obtained by carefully tuning the composition of the liquid mixture until the suspended solid particles are nearly immobile. At the chosen composition, particles rise within the fluid at a very slow speed of $4 \mathrm{~mm} / \mathrm{min}$. Using Stokes' law, this implies that the density difference ratio $\Delta \rho / \rho$ is down to about 6 parts per 10,000 . At this composition, the kinematic viscosity of the liquid is $v=37.2 \mathrm{~mm}^{2} / \mathrm{s}$.

Before an experiment, the particles are plunged into the liquid-filled chamber. The upside down conveyor belt system is then placed on top of the cavity. Before starting the flow, sufficient time elapses to let perturbations die down and allow the solid particles to rise up until they touch the lid. The particles are coloured dark blue and found easiest to image using back-illumination. Their motions are filmed under two different viewpoints, respectively, through the back wall and the side wall of the inner cavity. This is done using a single camera supplemented by a system of mirrors. The imaging sensor used is a TRV950 Sony DV camera operating in interlaced mode at a frequency of $30 \mathrm{~Hz}$. Images have a resolution of $480 \times 640$ pixels.

The camera selected allows compression-free acquisition of digital images over long durations. This permits continuous taping of each experiment, spanning both the sudden start-up of the conveyor belt and the long time regime established after many cycles. Lines of known interdistance are marked on the conveyor belt, allowing the belt speed to be monitored as well on the video. The belt is smooth and tightly stretched in order 
Fig. 1 Experimental setup: a overall view; b sketch of the imaging configuration including DV camera, mirror system and back-illumination rig; c side view of the lid-driven cavity; d front view

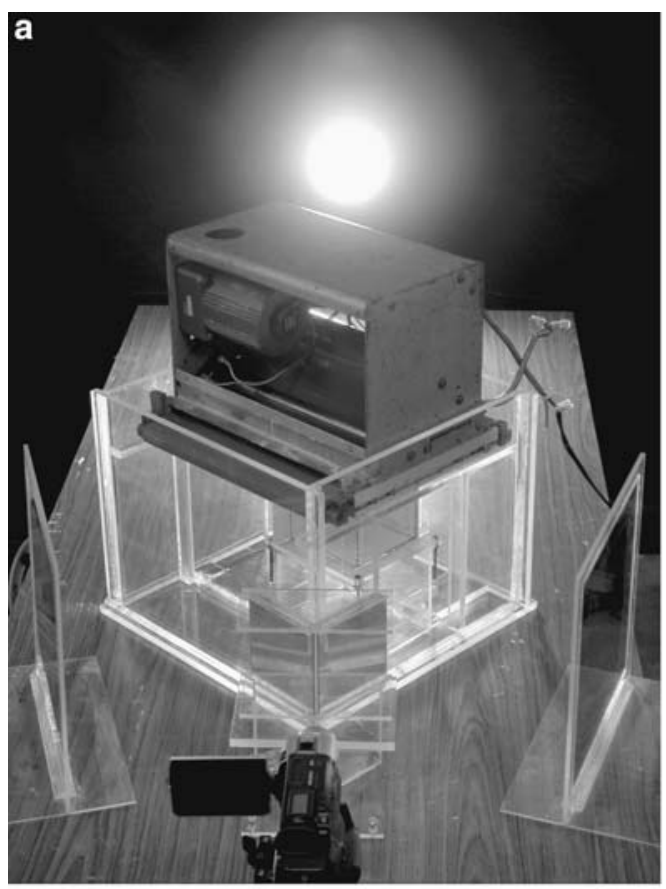

b
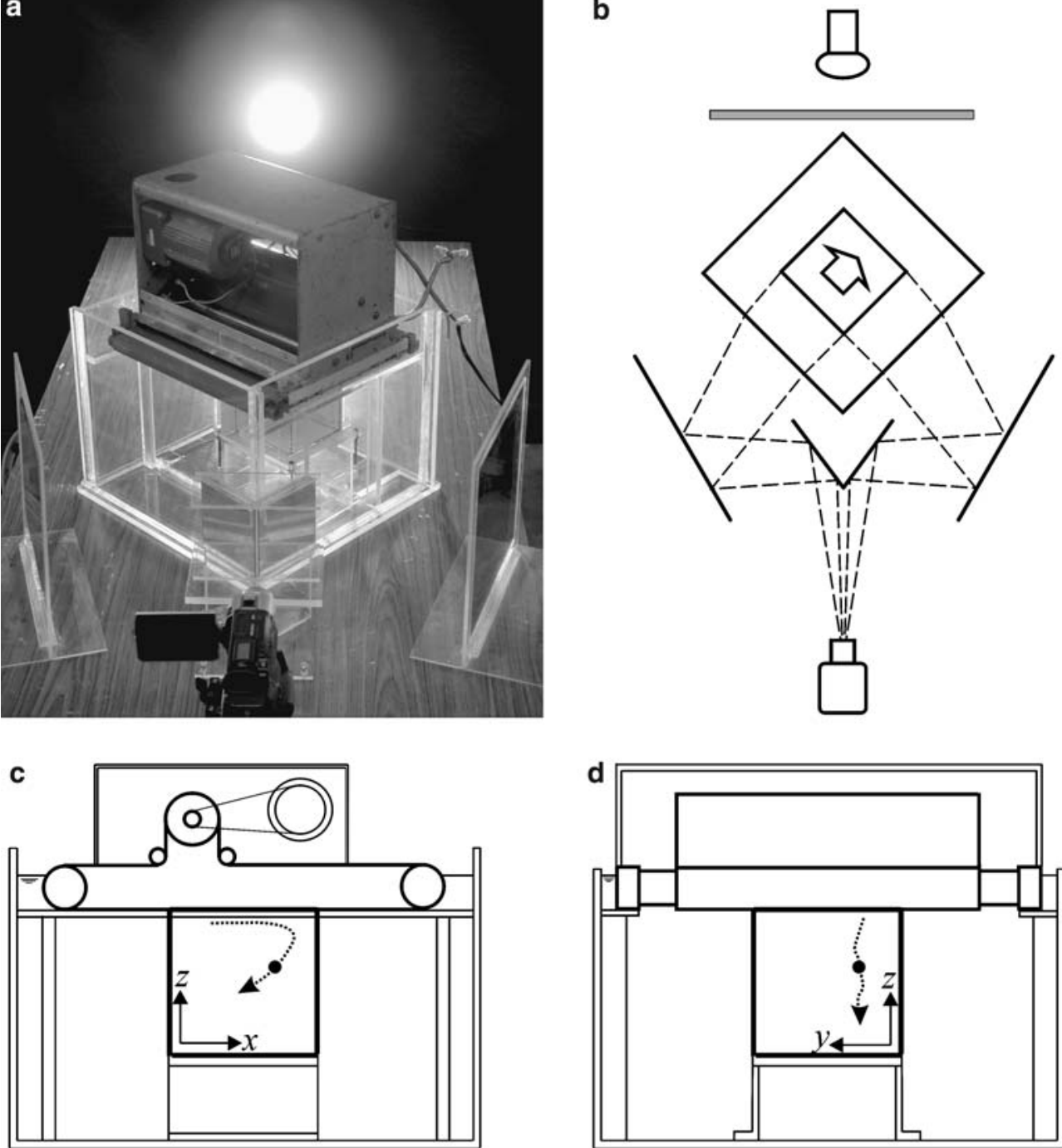

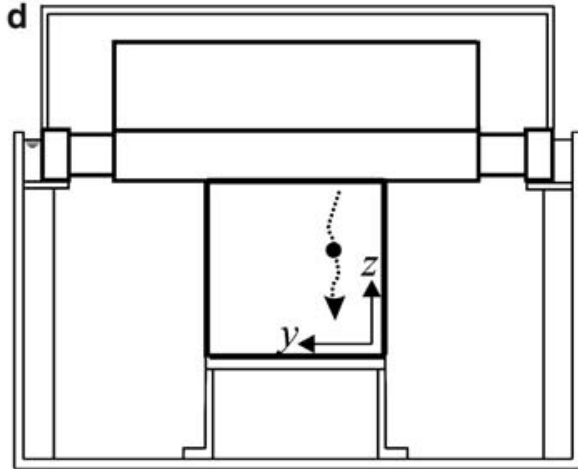

to avoid waviness. The test case used below to illustrate the methods was obtained at a belt speed $V=17.5 \mathrm{~cm} / \mathrm{s}$ (accurate to within $1 \mathrm{~mm} / \mathrm{s}$ ), corresponding to a cavity Reynolds number $R e=V S / v=470$. The total recording duration for this run was $7 \mathrm{~min}$. Further details of the lid-driven cavity device and imaging configuration are illustrated in Fig. 1.

\section{Particle positioning}

\subsection{Particle positioning on the $2 \mathrm{D}$ images}

The image analysis is performed entirely off-line. Once retrieved, the digital images are de-interlaced. This yields images of twice the frequency (60 instead of $30 \mathrm{~Hz}$ ), but half the vertical resolution (240 instead of 480 pixels). Image treatment then proceeds in the following steps. First, the gray scale is inverted to transform the backilluminated dark particles into bright spots over a dark backdrop. Next, a background image is obtained by averaging over the full image sequence. Difference ima- ges are then obtained by subtracting the background from each frame. This makes it easier to distinguish moving particles from static features of the images.

Standard methods are used to locate particles on these different images. Each image frame is first convoluted with a Laplacian-of-Gaussian mask (Jain et al. 1995) to highlight the bright particles of known pixel size. Local brightness maxima with peak values beyond a certain threshold are selected as particle centroids, and their positions are refined to subpixel accuracy using a quadratic fit. For more details, the reader is referred to Capart et al. (2002).

To make these procedures more robust, dynamic thresholding is used in the present work. Instead of choosing a constant threshold for particle identification, the threshold is set anew for each frame based on the previously observed brightness maxima. Typically the threshold is scaled down to two-thirds of the brightness maximum of the least conspicuous particle on the previous frame. This makes it easier to track particles over long time, during which they may gradually transit through regions of uneven illumination. The back-illu- 
mination is not perfectly uniform because of light reflection and refraction, as well as slight variations in translucency of the plates placed behind the cavity.

Results obtained using the above methods are illustrated in Fig. 2. Each panel shows a stereo view of the trajectories of two suspended solid particles, with the front view on the left and the side view on the right. For comparison purposes, panel a of Fig. 2 shows an artificial long exposure image, obtained simply by recording the brightest pixel of a sequence at each position. This highlights trajectories without recourse to any image analysis. For the same sequence, panel $b$ shows the marked particle positions acquired using the above algorithms. Overall, the captured particle positions align closely with the long exposure tracks. A small proportion of wrongly positioned particles is nevertheless present and will have to be dealt with at later stages of the analysis. Such mispositioning occurs when dark pixels of the images are mistaken for particles.

\subsection{Three-dimensional stereo positioning}

Once particles have been positioned on images from both viewpoints, their positions in 3D space can be found by locating the intersections of stereoscopic rays. As illustrated in Fig. 3, rays associated with two different viewpoints can be written in the parametric form:
Fig. 2 Particle positioning in the image plane: a artificial long exposure image obtained from a sequence of 410 frames;

b particle positions associated with the front view $(+)$ and side view $(\times)$, corresponding, respectively, to the left and right halves of the image. Two solid particles are present in this experiment

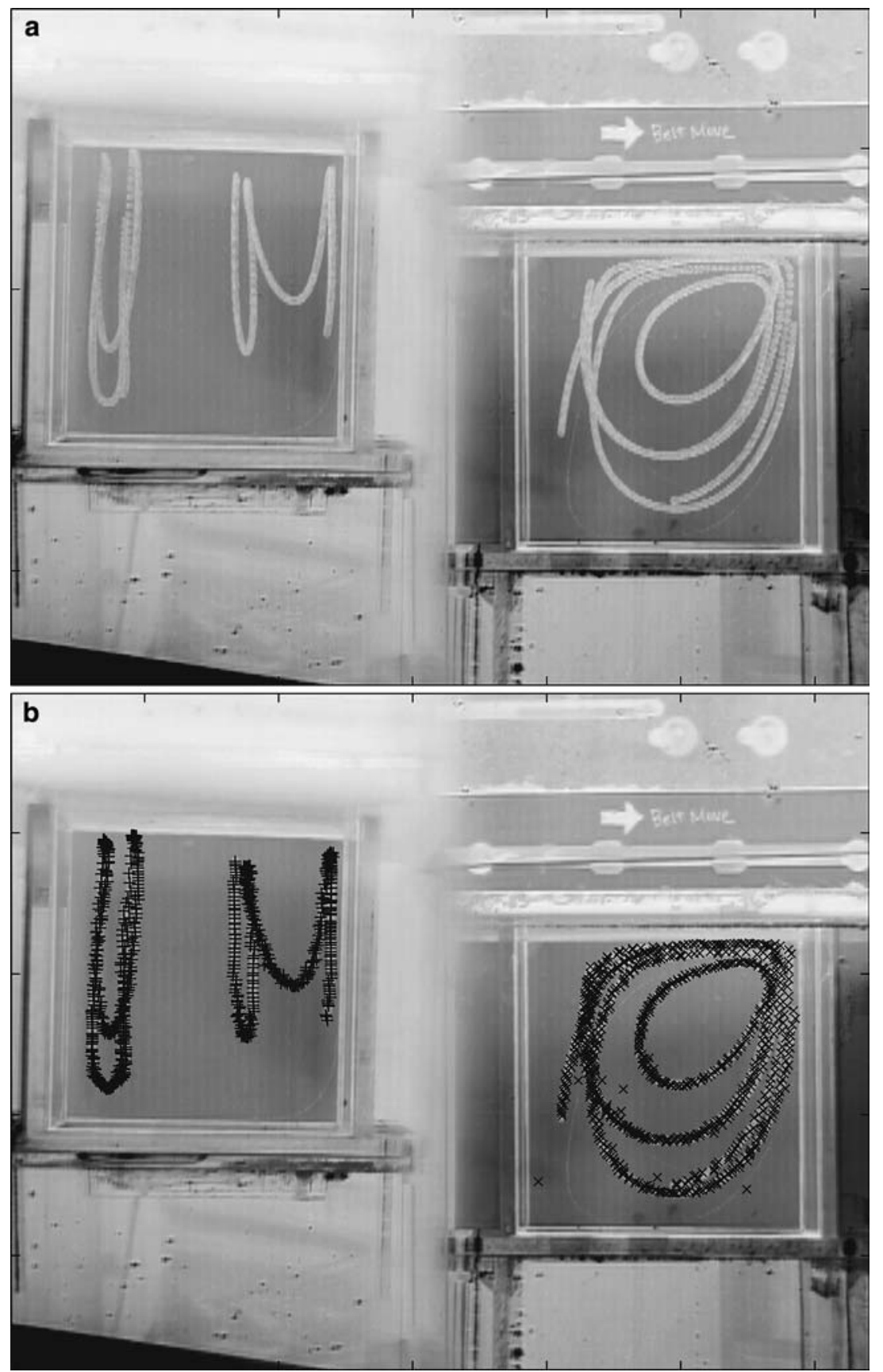


$\mathbf{x}(\lambda)=\mathbf{p}+\lambda \mathbf{q}$

$\mathbf{x}^{\prime}\left(\lambda^{\prime}\right)=\mathbf{p}^{\prime}+\lambda^{\prime} \mathbf{q}^{\prime}$,

where vector $\mathbf{x}=(x, y, z)=\left(x_{1}, x_{2}, x_{3}\right)$ denotes a position in $3 \mathrm{D}$ space, vector $\mathbf{p}=\left(p_{1}, p_{2}, p_{3}\right)$ denotes the $3 \mathrm{D}$ position of the projection centre (or focal point) of the first viewpoint, vector $\mathbf{q}=\left(q_{1}, q_{2}, q_{3}\right)$ gives the ray direction and scalar $\lambda$ is the free parameter. The same symbols marked with a prime denote corresponding entities for the second viewpoint.

The free parameters $\lambda$ and $\lambda^{\prime}$ are due to the depth ambiguity associated with 2D images: in the absence of other information, the location of a particle along a given ray is not known. This ambiguity must be resolved by using information from two different viewpoints. Provided rays from two such viewpoints are associated with one and the same physical particle, the $3 \mathrm{D}$ position of the particle will lie at the intersection of the rays. In practice, accuracy limitations prevent rays from perfectly intersecting each other. Instead, one seeks an approximate intersection point, defined as the midpoint of the shortest line segment linking the two rays (see Fig. 3). The parameters $\lambda, \lambda^{\prime}$ specifying the endpoints of this segment on each ray are the solutions of the linear system

$$
\left(\begin{array}{cc}
\mathbf{q}^{\mathrm{T}} \mathbf{q} & -\mathbf{q}^{\mathrm{T}} \mathbf{q}^{\prime} \\
\mathbf{q}^{\mathrm{T}} \mathbf{q} & -\mathbf{q}^{\prime} \mathbf{q}^{\prime}
\end{array}\right)\left(\begin{array}{c}
\lambda \\
\lambda^{\prime}
\end{array}\right)=\left(\begin{array}{c}
\mathbf{q}^{\mathrm{T}}\left(\mathbf{p}^{\prime}-\mathbf{p}\right) \\
\mathbf{q}^{\mathrm{T}}\left(\mathbf{p}^{\prime}-\mathbf{p}\right)
\end{array}\right),
$$

which states that the shortest line segment must be perpendicular to both rays. In this equation as well as other equations below, vectors are taken as column arrays and superscript $\mathrm{T}$ denotes the transpose. The mid-

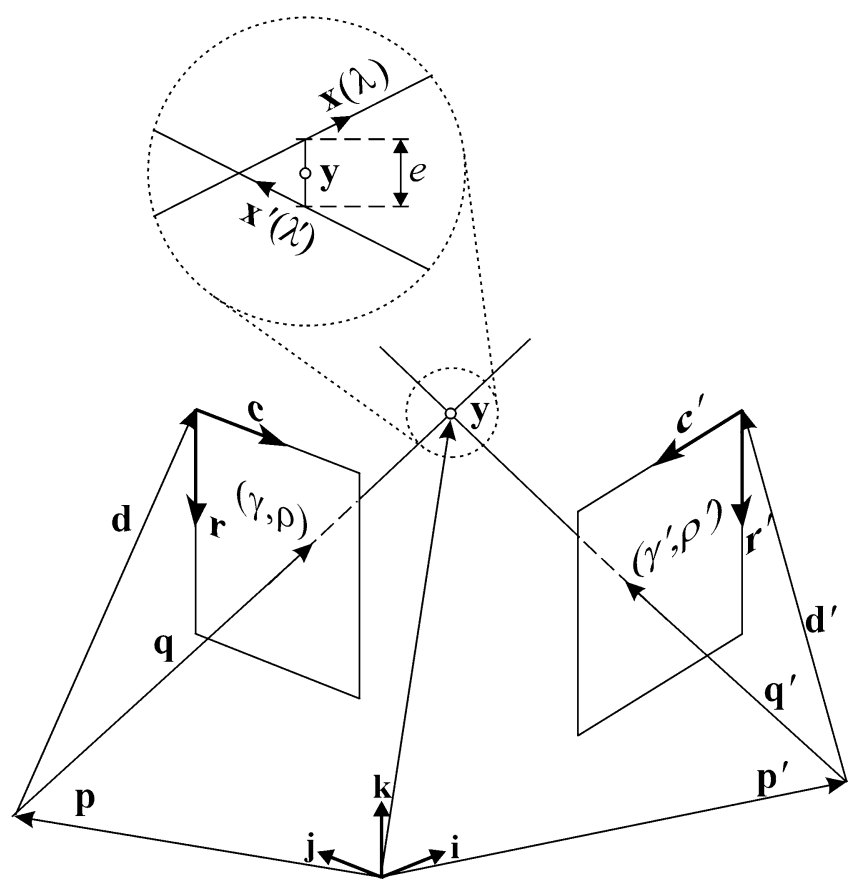

Fig. 3 Three-dimensional vector geometry of the stereo imaging configuration point position and distance of closest encounter are then given by

$\mathbf{y}=\frac{1}{2}\left\{\mathbf{x}(\lambda)+\mathbf{x}^{\prime}\left(\lambda^{\prime}\right)\right\}$

$e=\left\|\mathbf{x}^{\prime}\left(\lambda^{\prime}\right)-\mathbf{x}(\lambda)\right\|$.

Position $\mathbf{y}$ provides an approximation of the true position of the particle, while the distance $e$ provides an indication of the closeness of the intersection. When many particles are present, pairs of rays from the two viewpoints are selected by minimising the sum of their inter-ray distances, using the methods detailed in Spinewine et al. (2003). Once ray pairs have been obtained, their inter-ray distances also provide a measure of the accuracy of the stereoscopic reconstruction. This will be exploited below to estimate particle positioning errors.

\subsection{Camera calibration}

The parametric ray equations 1 and 2 are related as follows to the pixel coordinates of particles in each image plane. The image formation process is modelled as a perspective projection. As illustrated in Fig. 3, direction vector $\mathbf{q}$ can then be decomposed as the sum

$\mathbf{q}=\mathbf{d}+\gamma \mathbf{c}+\rho \mathbf{r}$,

where $(\gamma, \rho)$ are the (column, row) pixel coordinates of the particle centroid, depth vector d denotes the position of the origin of the image plane relative to the projection centre $\mathbf{p}$ and vectors $\mathbf{c}$ and $\mathbf{r}$ are direction vectors of the image plane associated, respectively, with column and row directions of the digital images. Equation 6 can be read as a parametric plane equation in which the two free parameters are the pixel coordinates $(\gamma, \rho)$. The parametric ray equation 1 can then be rewritten in the matrix form:

$\mathbf{x}(\lambda)=\mathbf{p}+\lambda\left(\begin{array}{ccc}\vdots & \vdots & \vdots \\ \mathbf{c} & \mathbf{r} & \mathbf{d} \\ \vdots & \vdots & \vdots\end{array}\right)\left(\begin{array}{l}\gamma \\ \rho \\ 1\end{array}\right)=\mathbf{p}+\lambda \mathbf{S}\left(\begin{array}{c}\gamma \\ \rho \\ 1\end{array}\right)$,

where the screen matrix $\mathbf{S}$ has vectors $\mathbf{c}, \mathbf{r}, \mathbf{d}$ as columns. Provided that projection centre $\mathbf{p}$ and screen matrix $\mathbf{S}$ are known, relation 7 transforms pixel coordinates on a $2 \mathrm{D}$ image into a parametric ray equation in $3 \mathrm{D}$ space. By marking each symbol with a prime, similar equations can of course be written for the second viewpoint.

The issue that remains to be addressed is the problem of estimating parameters $\mathbf{p}, \mathbf{S}$ and $\mathbf{p}^{\prime}, \mathbf{S}^{\prime}$ for the two viewpoints. This is the camera calibration problem, which can be solved by first acquiring images of calibration markers of known positions placed in the viewing volume of interest. The knowledge of at least six points of known image (2D) and world (3D) coordinates is sufficient to obtain the three components of vector $\mathbf{p}$ 
and the nine components of matrix $\mathbf{S}$. For this purpose, relation 7 is first inverted to obtain

$\lambda\left(\begin{array}{l}\gamma \\ \rho \\ 1\end{array}\right)=\mathbf{A}\left(\begin{array}{l}x \\ y \\ z\end{array}\right)+\mathbf{b}$,

where $\mathbf{A}=\mathbf{S}^{-1}$ and $\mathbf{b}=-\mathbf{S}^{-1} \mathbf{p}$. This relation can be observed to be linear in all the unknowns: $\lambda, a_{i j}$ and $b_{j}$. A stacked system of linear equations can thus be assembled with coefficients given by the known 2D and 3D coordinates $(\gamma, \rho, x, y, z)$ of each calibration point. A minor complication is that the resulting system is homogeneous and admits a trivial solution in which all unknowns are zero. This hurdle is easy to overcome by setting one of the unknowns equal to an arbitrary non-zero value. For six or more calibration points, the system is overdetermined and can be solved by linear least squares. The coefficients of $\mathbf{p}$ and $\mathbf{S}$ can finally be obtained from the coefficients of $\mathbf{A}$ and $\mathbf{b}$ using straightforward linear algebra. In practice, it is advised to choose more than six points, well distributed in the viewing volume of interest. In the present experiments, 12 points are used: the 8 corners of the cubic inner cavity supplemented by 4 points in a diagonal plane. For stereo imaging, the procedure must of course be applied to both viewpoints. More details about camera calibration can be found in Spinewine et al. (2003).

Once the two viewpoints have been calibrated, stereo rays can be used to find $3 \mathrm{D}$ particle positions using the method of Sect. 3.2. Figure 4 shows the corresponding results for the particles depicted earlier in Fig. 2. Qualitatively, the observed 3D trajectories are consistent with the information that can be visually inferred from the $2 \mathrm{D}$ views of Fig. 2. To assess the results quantitatively as

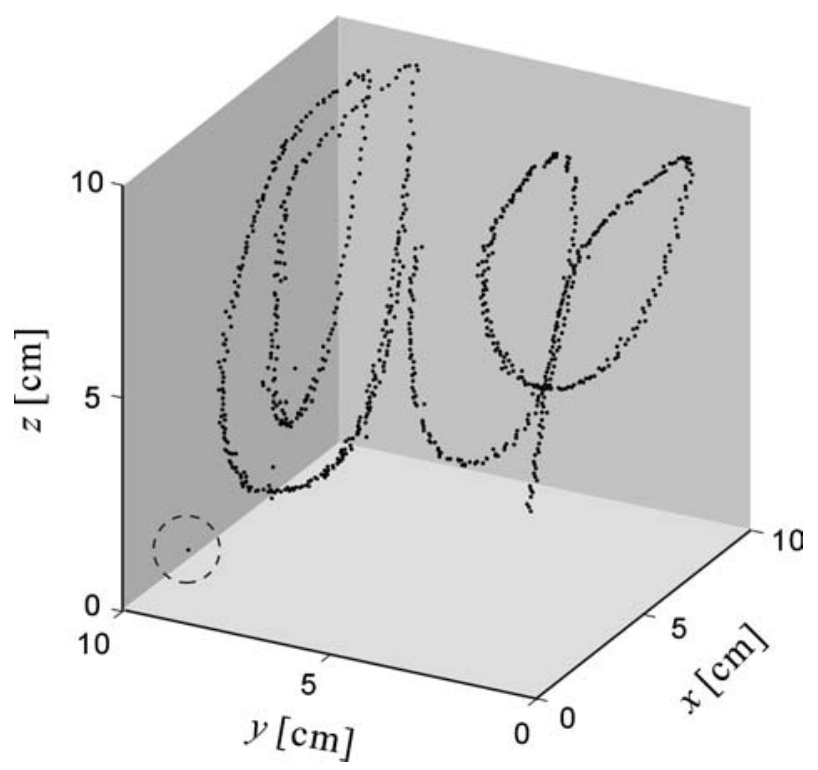

Fig. 4 Three-dimensional positions of the two particles of Fig. 2, obtained by stereo ray matching. A conspicuous mismatched outlier is circled near the lower left hand corner well, positioning errors associated with the stereo procedure are examined in the next section.

\subsection{Error estimation}

The errors incurred by the above procedure can be split into two classes: (1) a small proportion of misidentified particles and (2) measurement inaccuracies affecting correctly identified particles. Particles can be misidentified at both 2D and 3D stages: spurious features can be wrongly identified as particles on the $2 \mathrm{D}$ images (see Fig. 2) or stereo mismatches can occur when more than one particle is present in the viewing volume.

In the present work, the number of such mistakes is kept relatively low (compared for instance with the work reported by Spinewine et al. 2003 and Douxchamps et al. 2005), thanks to two features of the present experiments. First, motions are observed in a cavity with five transparent sides, allowing good control of the illumination conditions. Secondly, experiments are performed with only a limited number of particles, going from one to a maximum of 10 particles. To screen out misidentified particles from the data set, a simple outlier filtering procedure is used. For a given particle, positions which exceed a moving average position by more than four times a median absolute deviation are excluded from the set. Outliers screened out in this way are circled in Fig. 5. For the present experiment, this leads to the exclusion of less than $3 \%$ of the data.

On the other hand, the random position errors affecting correctly identified particles are estimated as follows. In the present experiments, the optical axes of the two viewpoints are nearly orthogonal to each other and lie in a horizontal plane. This means that 3D
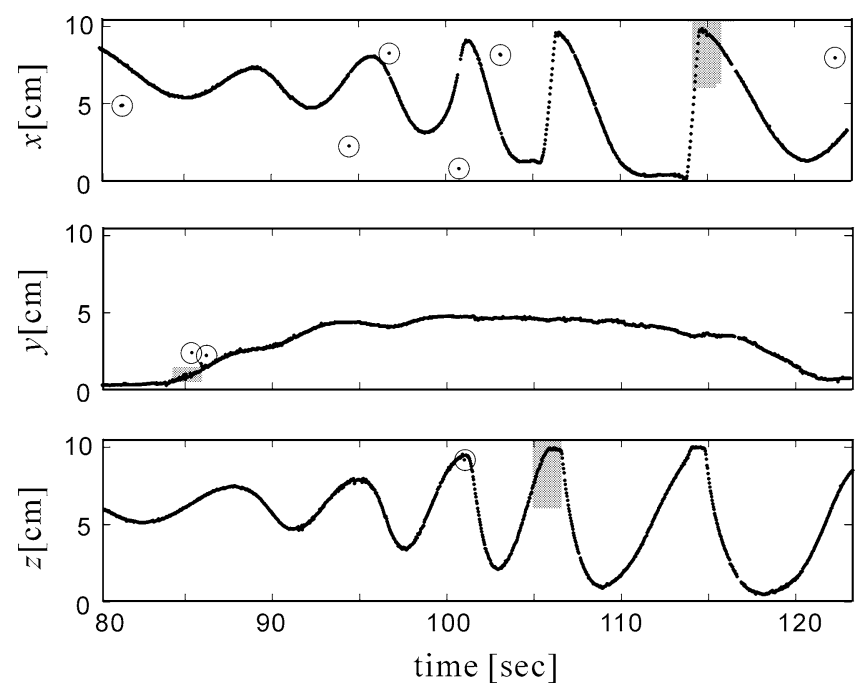

Fig. 5 Three-dimensional motions of a single suspended particle in the lid-driven cavity flow. The three panels show time series of the $x$-, $y$ - and $z$-coordinates, respectively, as measured by the stereo methods (before Kalman filtering). Grey boxes outline zones examined in detail in Figures 7 and 8 
position errors are simple to relate to $2 \mathrm{D}$ positioning errors on the digital images. Due to de-interlacing, the pixel resolution in the vertical (row) direction is half the resolution in the horizontal direction (column). Rootmean-squared (rms) position errors in the row and column directions are thus expected to follow the ratio

$\varepsilon_{r}=2 \varepsilon_{c}$,

where both are expressed in column pixel units. The corresponding position errors in $3 \mathrm{D}$ space are given by

$\varepsilon_{x}=\varepsilon_{y}=\alpha \varepsilon_{c}, \varepsilon_{z}=\frac{\alpha}{\sqrt{2}} \varepsilon_{r}$,

where $\alpha$ is a scale factor and the $\sqrt{2}$ denominator is an error attenuation produced by the average used to estimate midpoint (Eq. 4), under the assumption that random position errors are identically distributed and uncorrelated. This error attenuation is only operative in the $z$-direction, which corresponds to the row direction of both viewpoints. Likewise, the inter-ray distance (Eq. 5) is related to the row error through

$e=\alpha \sqrt{2} \varepsilon_{r}$.

Both the scale factor $\alpha$ and the inter-ray distance $e$ can be measured in the experiments. Based on the calibrated viewpoints, the scale factor is approximately $\alpha \approx 0.3 \mathrm{~mm} /$ (column pixel). Using the full set of ray pairs acquired in a typical experiment, on the other hand, the rms interray distance is of the order $e=0.6 \mathrm{~mm}$. Using Eqs. 9, 10 and $11, \mathrm{rms}$ errors for the raw stereo measurements of the 3D particle positions are found to be of the order

$\varepsilon_{x}=\varepsilon_{y}=0.2 \mathrm{~mm}, \quad \varepsilon_{z}=0.3 \mathrm{~mm}$,

and a combined position error can be obtained from

$\varepsilon=\sqrt{\varepsilon_{x}^{2}+\varepsilon_{y}^{2}+\varepsilon_{z}^{2}}=0.4 \mathrm{~mm}$.

The corresponding rms pixel error is $\varepsilon_{\mathrm{c}}=0.7$ pixel. This is slightly higher than typical errors reported in 2D PTV experiments (Veber et al. 1997; Capart et al. 2002). Note, however, that the error estimate obtained here includes both $2 \mathrm{D}$ image positioning errors and $3 \mathrm{D}$ geometrical effects (limited accuracy of the camera calibration and possible departures from the assumed perspective projection). As explained in the next section, the error level can be reduced further by applying Kalman filters to the particle trajectories.

\section{Kalman filtering of the particle trajectories}

\subsection{Measurement and signal models}

The stereo methods described above yield sets of $3 \mathrm{D}$ particle positions extracted from sequences of images. Long time trajectories are then constructed by linking together successive particle positions. When more than one particle is present in the cavity, a criterion must be invoked to decide which particle at one instant corresponds to which particle at the next instant. Since the present experiments involve small numbers of particles (maximum 10) embedded in a relatively regular flow, this particle tracking task is not difficult to carry out. A simple nearest-neighbour scheme (see, e.g. Guler et al. 1999) is used, subject to the condition that a particle can participate in only one trajectory (see Capart et al. 2002). The trajectory of each particle yields Lagrangian time series of raw position measurements. Such time series are shown in Fig. 5 for the three evolving spatial coordinates of a single particle.

To fill in data gaps and attenuate the effect of measurement noise, Kalman filtering can now be applied to the 3D position history of a given particle. The approach is based on an idealised model of the measurement and physical processes. The measurement model is simply

$\mathbf{y}_{k}=\mathbf{x}_{k}+\mathbf{m}_{k}$,

where vector $\mathbf{x}_{k}$ denotes the true $3 \mathrm{D}$ particle position $\left(x_{1}\left(t_{k}\right), x_{2}\left(t_{k}\right), x_{3}\left(t_{k}\right)\right)$ at time $t_{k}$, vector $\mathbf{y}_{k}$ denotes the measured particle position at the same instant and $\mathbf{m}_{k}$ represents the corresponding measurement noise. On the other hand, the physical particle trajectory is assumed to be governed by the following discrete signal model:

$$
\left(\begin{array}{l}
\mathbf{x}_{k} \\
\mathbf{u}_{k}
\end{array}\right)=\mathbf{F}\left(\begin{array}{l}
\mathbf{x}_{k-1} \\
\mathbf{u}_{k-1}
\end{array}\right)+\left(\begin{array}{c}
\mathbf{0} \\
\mathbf{n}_{k-1}
\end{array}\right)
$$

where vector $\mathbf{u}_{k-1}$ denotes the particle velocity averaged from time $t_{k-1}$ to time $t_{k}$. In the above equation, the matrix $\mathbf{F}$ is given by

$\mathbf{F}=\left(\begin{array}{cc}\mathbf{I} & \Delta t \mathbf{I} \\ \mathbf{0} & \mathbf{I}\end{array}\right)$,

where $\mathbf{I}$ is the $3 \times 3$ identity matrix and $\Delta t=t_{k}-t_{k-1}$ is the time interval between successive observations. Matrix $\mathbf{F}$ represents the kinematic relation linking two successive states $\left(\mathbf{x}_{k-1} ; \mathbf{u}_{k-1}\right)$ and $\left(\mathbf{x}_{k} ; \mathbf{u}_{k}\right)$. This deterministic link is perturbed by adding to the velocity vector $\mathbf{u}$ a random signal noise $\mathbf{n}$.

The signal noise contribution accounts for the physical particle accelerations, which are not taken into account by the deterministic component of kinematic model (Eq. 15). It is conceptually distinct from the measurement noise $\mathbf{m}$ introduced earlier. The two noise contributions nevertheless share one simplifying feature: the components of both vectors $\mathbf{m}_{k}$ and $\mathbf{n}_{k}$ at each time index $k$ are assumed to be uncorrelated, normally distributed random variables of zero mean. Note that because signal noise is added to the velocities, the latter remain self-correlated for some time.

Upon defining the full state vector

$\mathbf{z}=\left(\begin{array}{l}\mathbf{x} \\ \mathbf{u}\end{array}\right)$

relations 14 and 15 can be rewritten more compactly as 
$\mathbf{y}_{k}=\mathbf{H z}_{k}+\mathbf{m}_{k}$,

$\mathbf{z}_{k}=\mathbf{F} \mathbf{z}_{k-1}+\mathbf{n}_{k-1}$,

where $\mathbf{H}=(\mathbf{I} \mid \mathbf{0})$. Vectors $\mathbf{n}_{k-1}$ now feature six components instead of three, but it is implied that the first three components are zero. The equations are now in standard form, facilitating the description of the filtering process.

The filter adopted is the well-known Kalman filter (Kalman and Bucy 1961; Brown and Hwang 1997; Welch and Bishop 2001), used to yield an optimal estimate $\tilde{\mathbf{x}}_{k}$ of the particle position history. In the next two sections, we first deal with the case in which there are no missing data, then describe the adjustments necessary when data gaps are encountered. The ability of the Kalman filter to deal with both cases constitutes a major advantage for the processing of particle tracks.

\subsection{Forward Kalman filter for data without gaps}

Assuming first that no data gaps are encountered, Kalman filtering is carried out as follows. The filter proceeds recursively, through repeated application of two successive steps: a time update and a measurement update. First, a time update is performed using

$\hat{\mathbf{z}}_{k}=\mathbf{F} \tilde{\mathbf{z}}_{k-1}$.

This first step uses deterministic matrix $\mathbf{F}$ to predict the state at the next time instant based on the previous filtered state $\tilde{\mathbf{z}}_{k-1}$. In the second step, the measurement $\mathbf{y}_{k}$ is used to revise the prediction. The measurement update is written as

$\tilde{\mathbf{z}}_{k}=\hat{\mathbf{z}}_{k}+\mathbf{K}_{k}\left(\mathbf{y}_{k}-\mathbf{H} \hat{\mathbf{z}}_{k}\right)$,

where $\mathbf{K}_{k}$ is the Kalman gain matrix. In this second step, the new filtered state is estimated by making a correction proportional to the discrepancy between the predicted state and the actual measurement $\mathbf{y}_{k}$. The weight given to the measurement relative to the prediction is governed by the Kalman gain matrix $\mathbf{K}_{k}$. This matrix is constructed to minimise the a posteriori error covariance

$\tilde{\mathbf{P}}_{k}=E\left[\left(\tilde{\mathbf{z}}_{k}-\mathbf{z}_{k}\right)\left(\tilde{\mathbf{z}}_{k}-\mathbf{z}_{k}\right)^{\mathrm{T}}\right]$.

The error covariance matrix itself is obtained by recursion. For each iteration, two successive steps are associated, respectively, with the time update and the measurement update:

$\hat{\mathbf{P}}_{k}=\mathbf{F} \tilde{\mathbf{P}}_{k-1} \mathbf{F}^{\mathrm{T}}+\mathbf{Q}$,

$\tilde{\mathbf{P}}_{k}=\left(\mathbf{I}-\mathbf{K}_{k} \mathbf{H}\right) \hat{\mathbf{P}}_{k}$.

The Kalman gain matrix $\mathbf{K}_{k}$ used in statements 21 and 24 is calculated from

$\mathbf{K}_{k}=\hat{\mathbf{P}}_{k} \mathbf{H}^{\mathrm{T}}\left(\mathbf{H} \hat{\mathbf{P}}_{k} \mathbf{H}^{\mathrm{T}}+\mathbf{R}\right)^{-1}$.

In the above formulas, matrices $\mathbf{R}$ and $\mathbf{Q}$ are the covariances of the measurement noise and physical noise, respectively. They are given by

$$
\begin{gathered}
\mathbf{R}=E\left[\mathbf{m}_{k} \mathbf{m}_{k}^{\mathrm{T}}\right]=\left(\begin{array}{ccc}
\varepsilon_{1}^{2} & 0 & 0 \\
0 & \varepsilon_{2}^{2} & 0 \\
0 & 0 & \varepsilon_{3}^{2}
\end{array}\right), \\
\mathbf{Q}=E\left[\mathbf{n}_{k} \mathbf{n}_{k}^{\mathrm{T}}\right]=\left(\begin{array}{cc}
\mathbf{0} & \mathbf{0} \\
\mathbf{0} & \sigma^{2} \mathbf{I}
\end{array}\right) .
\end{gathered}
$$

For the measurement noise, the variances $\varepsilon_{1}^{2}, \varepsilon_{2}^{2}$ and $\varepsilon_{3}^{2}$ characterising position errors in the three space directions were obtained earlier from an analysis of the stereo imaging procedure (see Eq. 12). In contrast, the variance $\sigma^{2}$ of the physical signal noise is unknown a priori. In the absence of more information, it is assumed to be the same for all three velocity components.

\subsection{Forward Kalman filter for data with gaps}

The above procedure must be modified when missing data are encountered. Such data gaps occur whenever a particle position has been missed (typically due to occlusion effects if many particles are present) or when a particle position has been rejected as an outlier. In the present work, such gaps are relatively rare due to the small number of particles. Over long times, however, they are bound to be encountered at some points along the trajectories. The Kalman filter is used in the following way to deal with these gaps.

The time update which constitutes the first step of the Kalman filter is not modified. As before, statements 20 and 23 are used to forecast the system state $\hat{\mathbf{z}}_{k}$ and a priori error covariance $\hat{\mathbf{P}}_{k}$ at time $k$, based on information from the past. The measurement update, however, cannot be performed if measurement $\mathbf{y}_{k}$ is not available. In that case, the predicted state $\hat{\mathbf{z}}_{k}$ and covariance $\hat{\mathbf{P}}_{k}$ are adopted without correction. Statements 21 and 24 are thus replaced by

$\tilde{\mathbf{z}}_{k}=\hat{\mathbf{z}}_{k}$,

$\tilde{\mathbf{P}}_{k}=\hat{\mathbf{P}}_{k}$.

In other words, the filter can do no better than rely on prediction alone. At the next time $k+1$, the same steps are repeated if the data gap continues. Alternatively, if measured data become available again, the second step of the filter reverts to statements 21 and 24 .

Synthetic data are used in Fig. 6 to illustrate the response of the Kalman filter with and without gaps. The synthetic data are produced by Monte Carlo simulations of Eqs. 14 and 15, for the case of a particle moving in the $x$-direction only. The following parameters are used: time interval $\Delta t=0.02 \mathrm{~s}$; standard deviation of the measurement noise $\varepsilon_{1}=0.8 \mathrm{~mm}$; standard deviation of the process noise $\sigma=1 \mathrm{~mm} / \mathrm{s}$. In Fig. 6a, the bold line shows the noiseless physical signal, while dots represent the noisy measurements. The thin dashed line represents the Kalman filter response in the absence of gaps, while the thin continuous line represents the response when a subset of the measured data (circled dots) has been removed. 

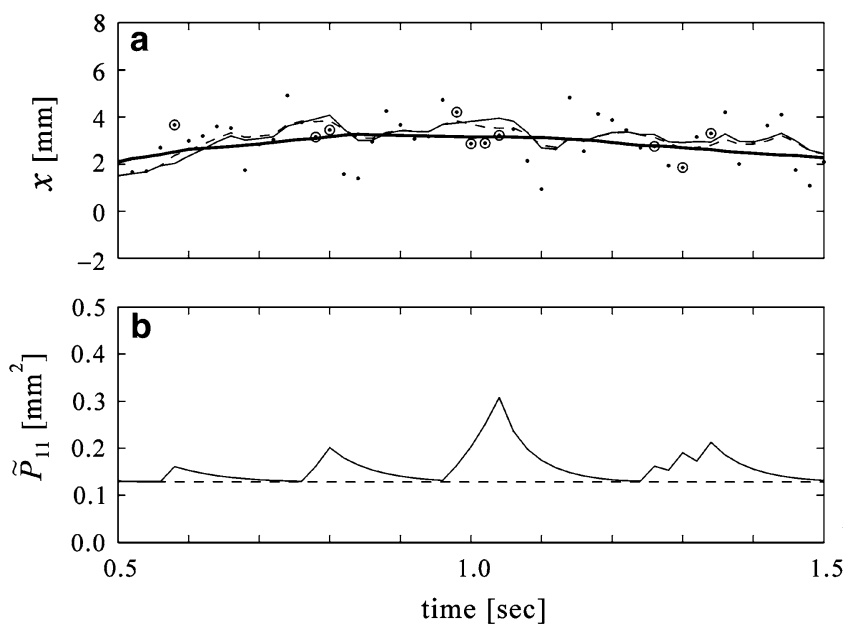

Fig. 6 Kalman filter response for synthetic data with and without gaps: a time series of position $x$ (thick line true position history; dots raw position measurements; dashed line filtered signal in the absence of data gaps; thin line filtered signal when the circled data points are missing); $\mathbf{b}$ variance of the position error (dashed line variance when no data gaps are present; continuous line variance when the circled data of a are missing)

Figure $6 \mathrm{~b}$ shows the a posteriori variance of the position error $\tilde{P}_{11}$ when gaps are absent (dashed line) and when gaps are present (continuous line). These values can be compared with the error variance of the raw data $\varepsilon_{1}^{2}=0.64 \mathrm{~mm}^{2}$. After transient initial adjustments have been made (not shown), the filtered positions have a constant error variance of $\tilde{P}_{11}=0.13 \mathrm{~mm}^{2}$ when there are no data gaps. In the presence of data gaps, in contrast, the variance grows each time a gap is encountered, then steadily diminishes when measured data become available again. Obviously, the accuracy of the filtered signal deteriorates when data gaps are encountered. Nevertheless, as in this example, the filtered signal obtained by processing gappy data can remain more accurate than the raw gapless signal. This illustrates how the Kalman filter can both attenuate errors and deal with missing data.

\subsection{Forward-backward Kalman filter}

In practice, results obtained with the above forward filtering pass are not entirely satisfactory. In addition to attenuating high-frequency noise, which is the desired outcome, the filter shifts the signal in time. As illustrated in Fig. 7, this effect is especially pronounced when a low signal variance $\sigma^{2}$ is assumed. A remedy is to apply both a forward and a backward pass to the signal and to blend their results (see Brown and Hwang 1997). In this case, let $\tilde{\mathbf{z}}_{k-1}$ denote the filtered state at time $t_{k-1}$ obtained by starting from $t_{1}$ and applying the Kalman filter forward in time. Matrix $\tilde{\mathbf{P}}_{k-1}$ denotes the corresponding error covariance. Steps 21 and 23 can then be used to perform the time updates

$\hat{\mathbf{z}}_{k}=\mathbf{F} \tilde{\mathbf{z}}_{k-1}$
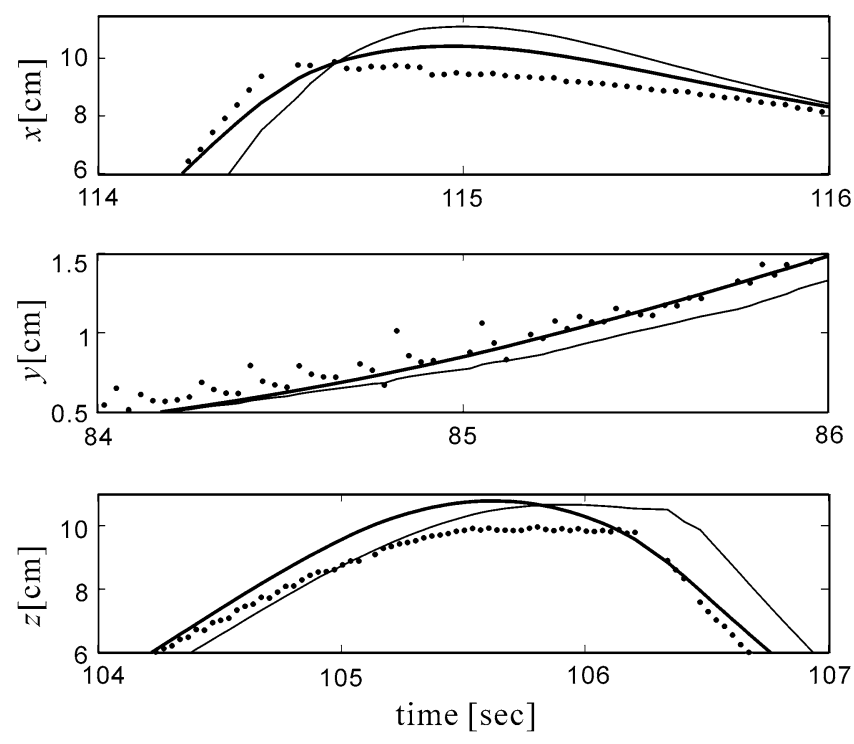

Fig. 7 Zoom on portions of the position time series of Fig. 5. Dots stereo data; thin line signal obtained after a single forward pass of the Kalman filter, exhibiting an undesirable time shift to the right; thick line signal resulting from a combination of forward and backward passes

and

$\hat{\mathbf{P}}_{k}=\mathbf{F} \tilde{\mathbf{P}}_{k-1} \mathbf{F}^{\mathrm{T}}+\mathbf{Q}$,

where hats indicate quantities obtained by forward prediction. If $n$ successive position measurements are available, a filtered state $-z_{k}$ and error covariance $\leftarrow P_{k}$ at time $t_{k}$ can likewise be obtained by starting from $t_{n}$ and applying the Kalman filter backward in time. A blended estimate can then be obtained from the weighted sum

$\leftrightarrow z_{k}=\left(\hat{\mathbf{P}}_{k}^{-1}+-P_{k}^{-1}\right)^{-1}\left(\hat{\mathbf{P}}_{k}^{-1} \hat{\mathbf{z}}_{k}+\leftarrow P_{k}^{-1} \leftarrow z_{k}\right)$,

which constitutes the result of the forward-backward filter. Note that by using time updates 29 and 30, the forward pass does not make use of measurement $\mathbf{y}_{k}$, which is used only by the backward pass. This is needed to avoid using the same information twice. Results obtained with this forward-backward version of the Kalman filter are also shown in Fig. 7. It is seen that the time shift has vanished, leaving only the desired signal attenuation.

\subsection{Filter tuning}

The degree of attenuation produced by the filter is governed by the relative strength of the measurement error and physical noise, gauged by the non-dimensional ratio $\beta=\sigma \Delta t / \varepsilon$. This ratio depends on both the known measurement error level $\varepsilon$ and the unknown physical noise strength $\sigma$. The last issue to be addressed is therefore the choice of physical noise strength $\sigma$, or equivalently the choice of ratio $\beta$ used to tune the filter. 

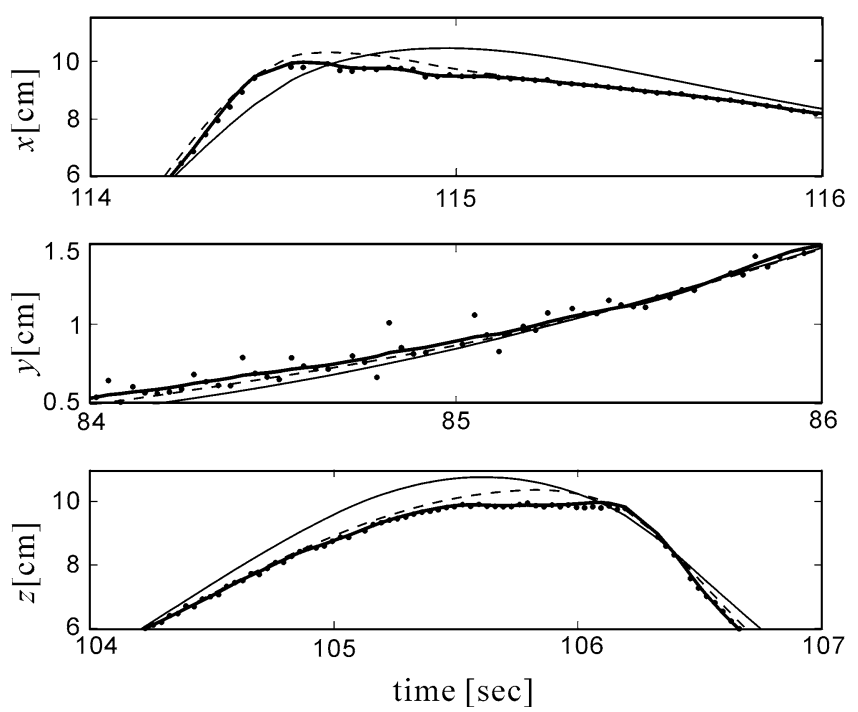

Fig. 8 Kalman filter results for different values of ratio $\beta=\sigma \Delta t / \varepsilon$ representing the strength of the measurement error noise relative to the physical signal noise. Dots raw data; thin line $\beta=0.005$; dashed line $\beta=0.025$; thick line $\beta=0.2$

As illustrated in Fig. 8, different values of $\beta$ lead to contrasted outcomes. High values of $\beta$ imply that the observed random jitter of the signal is mainly physical in origin and should not be attenuated. On the other hand, low values imply that most of the jitter is due to measurement noise and should be filtered out. The Kalman filter responds accordingly, attenuating the signal in inverse proportion to ratio $\beta$.

Since the true physical signal is unknown, it is a priori unclear how $\beta$ should be chosen. The approach adopted is to apply the forward-backward filter to the signal for various values of ratio $\beta$ and to monitor the meansquared difference between the resulting filtered positions $\leftrightarrow x_{k}(\beta)$ and the raw measured positions $\mathbf{y}_{k}$. In contrast to the random measurement noise, the physical position jitter is expected to be correlated in time. It is thus less sensitive to attenuation and we can assume that for the correct choice of $\beta$, the filtered positions $\leftrightarrow x_{k}(\beta)$ provide a reasonable approximation of the true signal $\mathbf{x}_{k}$. The mean-squared difference between $\rightarrow x_{k}(\beta)$ and $\mathbf{y}_{k}$ should then be close to the known measurement noise variance $\varepsilon^{2}$. This motivates the following rule of thumb: our choice for $\beta$ is the value which yields a meansquared difference between $\leftrightarrow x_{k}(\beta)$ and $\mathbf{y}_{k}$ equal to the known error variance $\varepsilon^{2}$. The criterion is illustrated in Fig. 9, and the resulting filtered signal is plotted as a thick line in Fig. 8.

\section{Results and discussion}

\subsection{Time series of particle positions}

Time series of the three components of the filtered position $\leftrightarrow x$ of a single particle (noted $x, y, z$ for simplicity

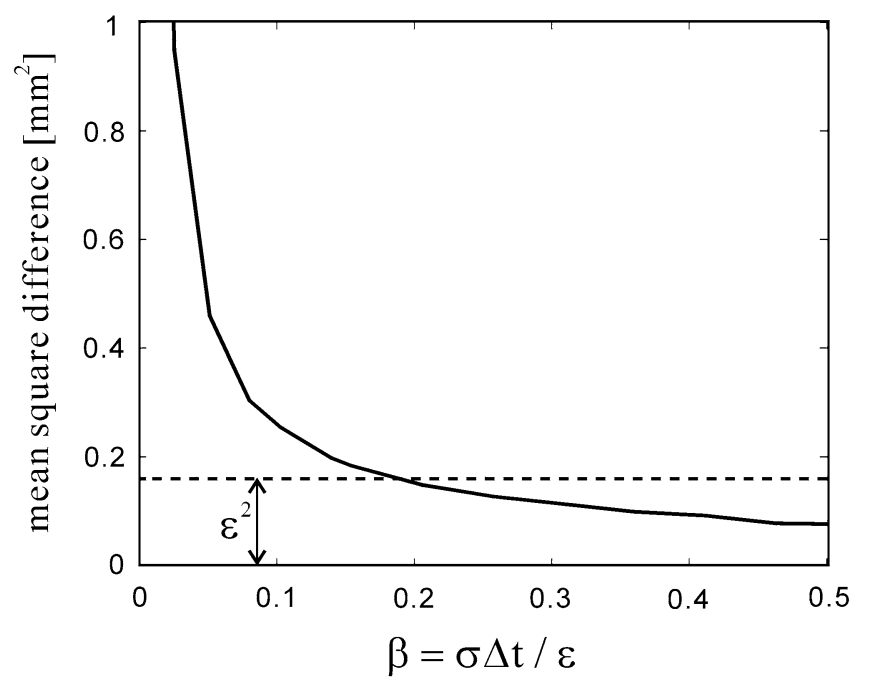

Fig. 9 Tuning criterion for ratio $\beta$ : the value selected is the one yielding a mean-squared difference between $\leftrightarrow x_{k}(\beta)$ and $\mathbf{y}_{k}$ equal to the known noise variance $\varepsilon^{2}$

in the legend) are plotted in Fig. 10. Here, the first $3 \mathrm{~min}$ of an overall measured span of $7 \mathrm{~min}$ is represented. Beyond the first minute, the observed motions stay similar in character for the rest of the sequence, indicating that a steady-state regime has been reached. Various features of the particle orbits can be observed on these plots.

Overall, the particle motions feature two main components: fast laps in the $x-z$ plane and slow sideways motions in the $y$-direction. First, the $x$ - and $z$-position histories exhibit fast oscillations of gradually varying amplitude and period, out of phase with each other by $90^{\circ}$. This implies spiral motions of the particle about a rotation axis parallel to the $y$-direction. These motions directly reflect the rotational flow induced by the longitudinally driven lid. The outermost excursions of the particle bring it close to the conveyor belt, located at plane $z=10 \mathrm{~cm}$. As the particle approaches the upstream edge of the belt (line $x=0, z=10 \mathrm{~cm}$ ), it makes a characteristic hairpin turn. Upon reaching the downstream edge (line $x=10 \mathrm{~cm}, z=10 \mathrm{~cm}$ ), it makes a sharp downward turn.

In addition to these fast laps around the cavity, the $y$ position history exhibits slow cycles of lateral motion. Rather than staying within a single plane, the particle swerves sideways. These slow lateral motions have large amplitudes, bringing the particle very near the centre plane $(y=5 \mathrm{~cm})$ and the right lateral wall of the cavity $(y=0 \mathrm{~cm})$. Similar motions (not shown) are observed for a second particle located to the left of the centre plane. At this Reynolds number, particles are not observed to ever cross the centre plane of the cavity. Comparing the different time histories with each other, it is clear that the evolution of the amplitude of the rapid $x-z$ cycles is modulated by the slow lateral motions. This modulation is not quite periodic. Instead, irregular cycles of variable duration are observed throughout the sequence. 

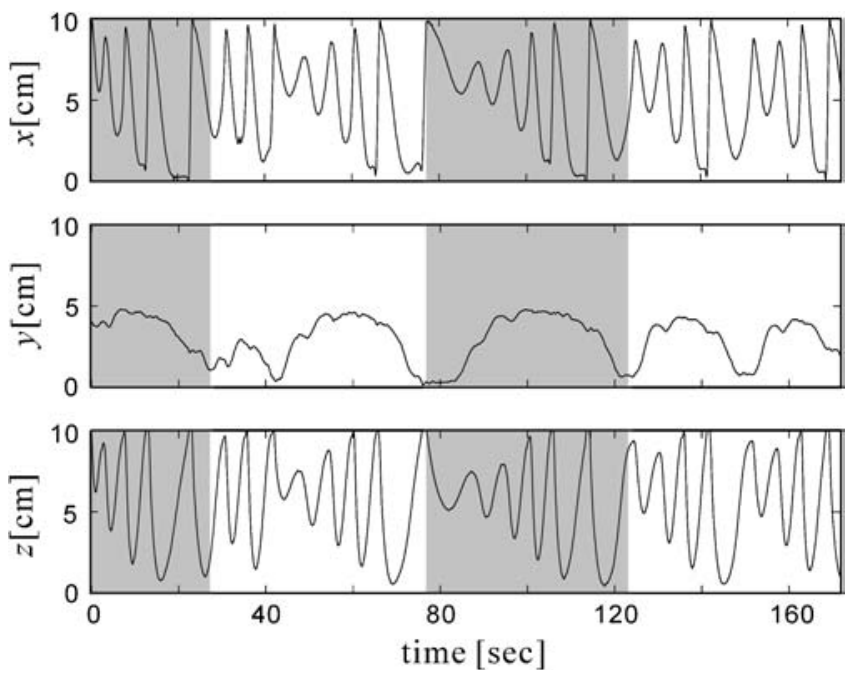

Fig. 10 Time series of the three components of the filtered position $\rightarrow x$ of a single particle. The shaded regions correspond to the startup and typical cycle regime segments examined in greater detail in the next figures

Nevertheless, a generic feature of these cycles is that the particle lingers near the centre plane, but makes only short-lived excursions to the neighbourhood of the sidewall. Other features of the particle trajectory are easier to understand based on 3D views, and are discussed in the next subsection.

5.2 Three-dimensional trajectory of an individual particle

For clarity, it is convenient to look separately at two shorter segments of the orbits, shaded in grey in Fig. 10: the start-up phase, immediately following the sudden start of the conveyor belt, and a typical cycle of the steady-state regime. Three-dimensional views of the corresponding trajectories are plotted in Figs. 11 and 12, respectively.

At the beginning of the start-up phase, the particle is located near the centre plane and along the top lid. This position is marked as a black dot on the four panels of Fig. 11. After the sudden start of the conveyor belt, the particle is entrained into circuitous orbits. At first, the orbit is tightly wound and confined to the downstream end of the cavity, but then gradually spirals outwards. Throughout these expanding orbits, the particle stays near the centre plane. Upon reaching the perimeter of the cavity, the orbit finally exhibits its first significant lateral drift, moving outwards towards the side wall (plane $y=0$ ) along the back face (plane $x=0$ ). This orbit corresponds to a transient phase of the liquid flow field.

A more complete orbital cycle is shown in Fig. 12. The cycle corresponds to a later time, after establishment of a steady-state regime of qualitatively recurrent behaviour. The specific cycle chosen, somewhat longer than typical cycles, was selected because it shows the orbital pattern particularly clearly. Starting from a position near the side wall of the cavity, the particle spirals inwards towards the centre plane. The spiral is at first tightly wound, but gradually expands as the centre plane is approached. Near the centre plane, the particle makes a few more laps, while continuing to slowly spiral outwards towards the perimeter of the cavity. Finally the particle drifts back towards the side wall along the back and bottom faces of the cavity (planes $x=0$ and $z=0$, respectively). Compared to the start-up phase, the axis of the spiral is closer to the centroid of the cavity, but remains in the upper downstream quadrant. Such particle trajectories spiralling inwards then outwards around the centreline of the main eddy have been obtained earlier from numerical computations of the liddriven cavity flow by Chiang et al. (1998) and Sheu and Tsai (2002).

\subsection{Comparison with laser-illuminated micro-particle} tracks

In order to further evaluate and interpret the observed trajectories of the solid particles, experiments at the same Reynolds number $R e=470$ were conducted with laser-illuminated micro-particles instead of full-volume stereo imaging. In these experiments, the liquid is seeded with PSP micro-particles having diameter equal to $50 \mu \mathrm{m}$. Illumination is then provided by a high-power laser light sheet having a thickness of approximately $2 \mathrm{~mm}$. For the results reported below, experiments were conducted with the light sheet oriented parallel to the side walls of the cavity and placed, respectively, at distances $y=2 \mathrm{~cm}$ and $y=4 \mathrm{~cm}$ from the right sidewall. To record the flow pattern within the illuminated plane, an AVT CCD camera was used. This camera has a resolution of $1,024 \times 768$ pixels and operates at a frame rate of $15 \mathrm{fps}$.

In order to characterise the Eulerian flow pattern at steady state, the cavity is operated at constant belt speed for some $20 \mathrm{~min}$ before images are acquired. Artificial long exposure images are constructed from sequences of images in the same way as before, by registering at each pixel position the brightest value experienced during the entire sequence. As they are associated with micro-particles convected in a steady-state flow field, the bright pathlines recorded in this way can be regarded as streamlines of the flow. These illuminated streamlines are shown in Fig. 13, where they are compared with partial trajectories of the $3 \mathrm{~mm}$ solid particles obtained using the stereo approach described earlier. For this purpose, 2D trajectory segments must be extracted from the complete $3 \mathrm{D}$ trajectories. This is done by retaining only the particle positions comprised within thin slices of half-thickness equal to $1.25 \mathrm{~mm}$ on both sides of the medial plane of the laser sheet. Black dots are used in Fig. 13 to represent the resulting subset of tracked particle positions.

The results of Fig. 13 show a close alignment of the solid particle paths with the laser-illuminated pathlines 
Fig. 11 Three-dimensional trajectory of a suspended solid particle in the lid-driven cavity flow $(R e=470)$. Segment of $23 \mathrm{~s}$ immediately following the sudden start-up of the conveyor belt. a Side view, b front view, c top view, d 3D plot

Fig. 12 Three-dimensional trajectory of a suspended solid particle in the lid-driven cavity flow $(R e=470)$. Typical orbital cycle over a span of 43 s. a Side view, b front view, c top view, d 3D plot
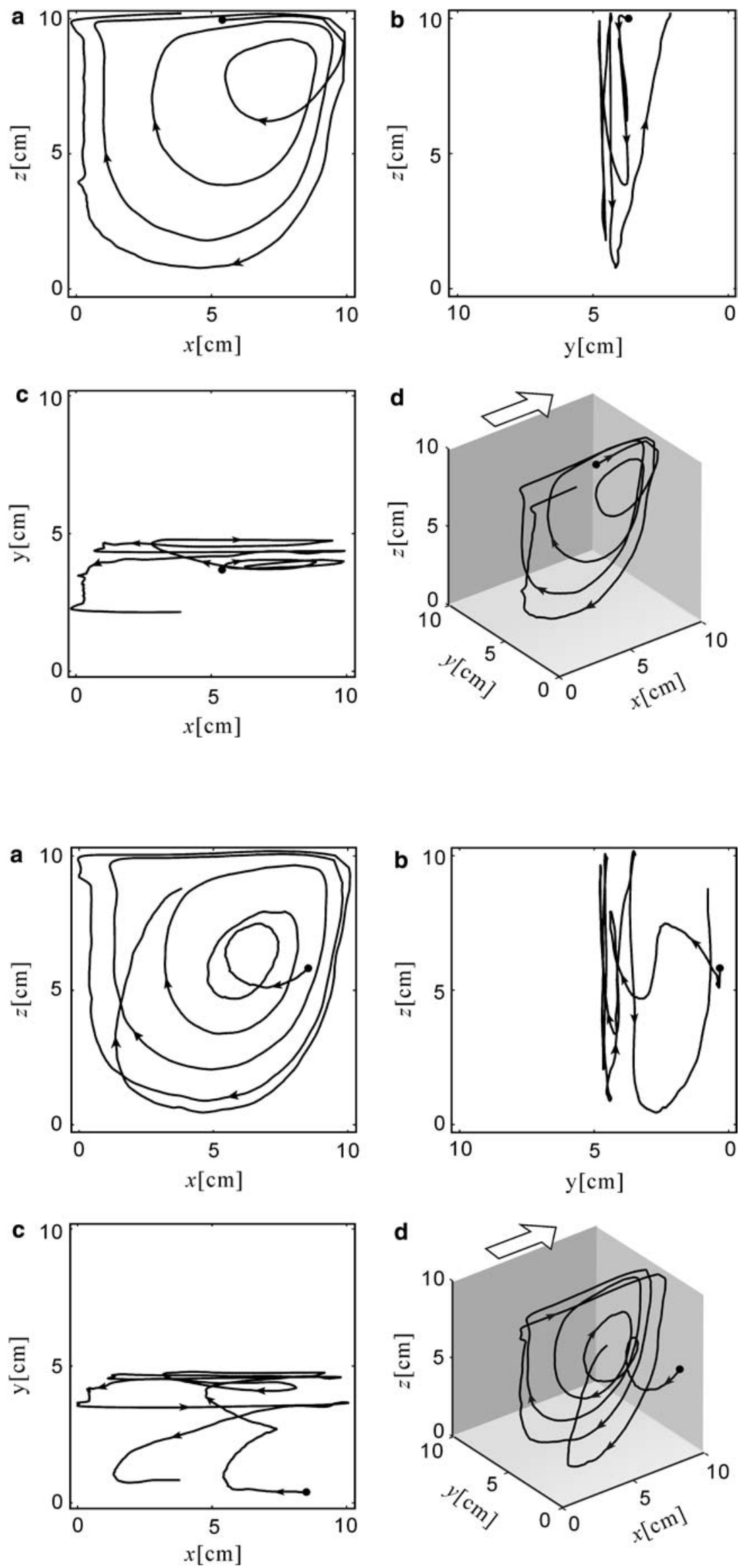
Fig. 13 Solid particle tracks (black dots) overlaid onto long exposure images of laserilluminated micro-particles at $R e=470:$ a transverse plane $y=2 \mathrm{~cm} ; \mathbf{b}$ transverse plane $y=4 \mathrm{~cm}$
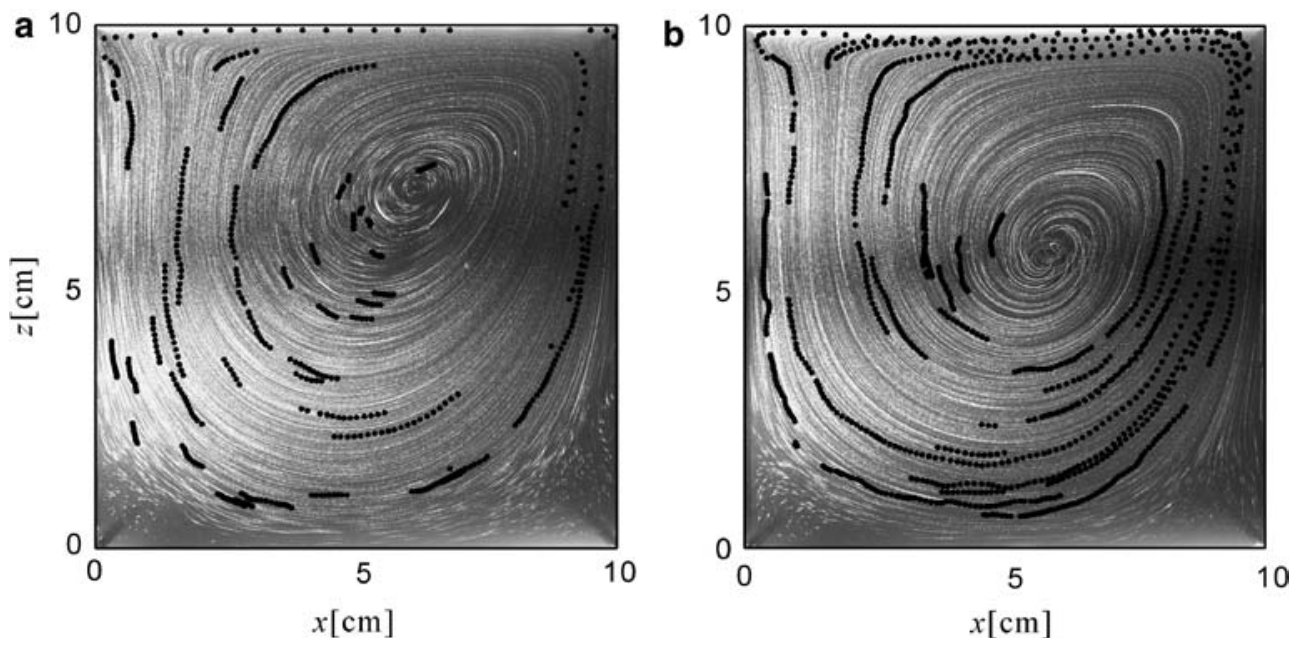

of the micro-particles. This indicates that the solid particle motions reflect rather faithfully the motions of the embedding fluid. The juxtaposition of these two independently produced results can also be used as a qualitative check of the methods. Encouragingly, jitter due to measurement noise is attenuated by the Kalman filters, without significantly distorting the shapes of the orbits.

\subsection{Trajectories of 10 orbiting particles}

The experimental run examined until now was a run conducted with two particles only, and attention was focused on the motions of only one of them. To demonstrate the wider applicability of the technique and highlight certain additional features, this last subsection presents results for a run involving 10 simultaneously orbiting particles. The corresponding Reynolds number is slightly higher at $R e=V S / v=1,030$. All 10 particles were initially placed in the right half of the cavity and stayed there for the entire run. Other runs conducted with particles on both sides showed a high degree of symmetry.

Figure 14 shows the orbits of the 10 particles, as tracked over 500 successive frames (a segment of $17 \mathrm{~s}$ in the steady-state regime after the start-up transient has died out). Panel a of Fig. 14 shows a stereoscopic long exposure image, while panels b-d show the corre-
Fig. 14 Simultaneous orbits of 10 suspended solid particles in the right half of the lid-driven cavity $(R e=1,030)$, over a span of $17 \mathrm{~s}$. a Long exposure image obtained from a sequence of 500 frames, b 3D plot, c front view, $\mathbf{d}$ side view
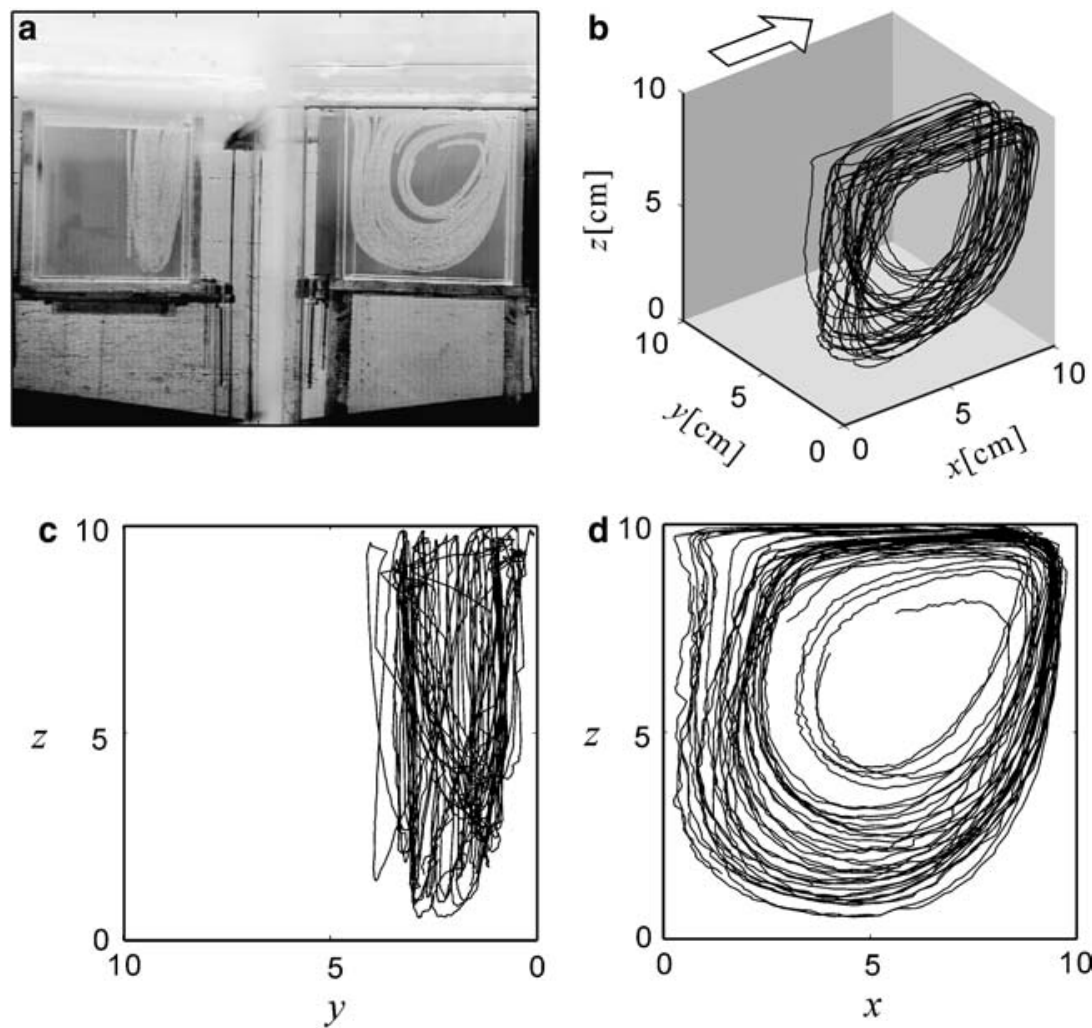
sponding 3D trajectories. Like the image of Fig. 2a, the long exposure view of Fig. 14a is produced directly from the video frames and does not rely on the positioning algorithms. In contrast, the curves plotted in panels b-d represent stereo measurements, after full processing by the forward-backward Kalman filters.

The results highlight an intriguing feature of the experiments. Despite starting from arbitrary positions and being allowed to wander freely within the cavity, the neutrally buoyant particles do not explore all regions of the flow. Instead, they tend to cluster along preferential pathways of the internal circulation: an inner coil along which particles spiral inwards from the sidewall towards the centre plane and an outer coil along which particles drift back from the centre plane towards the sidewall. Conversely, there are various zones in which the particles do not venture: (1) the core of the main vortex, near the centre of the cavity; (2) the corner eddies located at the four corners of the bottom face of the cavity; (3) a toroidal zone located between the inner and outer coils along which particles spiral inward and outward.

In contrast, the numerical computations reported by Chiang and Sheu (1997) indicate that fluid particle paths do link the central eddy to the upstream and downstream corner eddies. Over long time orbits, fluid particles should thus be able to explore the various connected regions of the 3D flow. It is currently unclear why the solid particles of the present experiments do not appear to behave in this manner. One possibility is that the observation time remains too short for the particles to be seen in all the zones that they can actually reach. Another is that the finite size particles cannot enter the very near-wall regions of the flow, which certain fluid pathways must go through. A third possibility is that the solid particles drift towards preferential orbits because of inertial migration, following a mechanism similar to the one observed in Poiseuille flows (Han et al. 1999; Matas et al. 2004). Further research is definitely needed to clarify this issue.

\section{Conclusions}

In the present work, stereo imaging and signal-processing techniques were combined to monitor the $3 \mathrm{D}$ orbits of individual particles in a lid-driven cavity flow. Using a digital camera, video sequences of long duration were acquired, continuously recording the particle motions under two different viewpoints. By tracing calibrated rays into the viewing volume, the $3 \mathrm{D}$ particle positions and corresponding measurement errors were estimated. To attenuate the effect of these errors, the position signals were then processed by Kalman filters, based on a simple stochastic model of the kinematics. Kalman filters with forward and backward passes were found to give good results, attenuating the noise without shifting the signals in time or significantly distorting the orbital shapes.
The measured particle trajectories present a number of interesting characteristics. Overall, the particles are observed to undergo spiral motions modulated by sideways excursions from the centre plane to the side wall and back. These spiral motions are found to exhibit slightly different patterns during the start-up phase and the subsequent regime. At steady state, the particle trajectories within selected longitudinal slices align with the pathlines of laser-illuminated micro-particles. Nevertheless, the solid particle orbits are observed to cluster along certain pathways of the internal circulation and avoid altogether certain regions of the cavity flow. The origin of this behaviour is currently unclear. Taken together, these observations carry some practical implications for PTV studies. Whereas the paths of neutrally buoyant solid particles may faithfully reflect the local motions of the fluid, there is no guarantee that these solid particles will be present in all zones of the flow. In the present experiments, for instance, the paths of the solid particles provide no information about the fluid motions within the secondary eddies of the cavity.

The research suggests various avenues for further work. The measurement methods could benefit from a number of improvements. Instead of using the Kalman filters at the post-processing stage only, they could be incorporated at the particle tracking stage. This could make the methods more robust, allowing them to deal with more difficult illumination conditions or larger numbers of simultaneously orbiting particles. More rigorous ways of tuning Kalman filters would also be desirable. Likewise, further work is needed to enhance our understanding of the motions of solid particles suspended in 3D viscous flows. In particular, the possible migration of the particles towards preferential zones requires further scrutiny. For the lid-driven cavity, we are currently engaged in efforts aimed to characterise particle trajectories over a wider range of Reynolds numbers and to further probe the relationship between the Lagrangian particle trajectories and the Eulerian viscous flow field.

Acknowledgements Support from the National Science Council, Taiwan, is gratefully acknowledged. Thanks are also conveyed to the two anonymous reviewers for their constructive and useful comments.

\section{References}

Adrian RJ (1991) Particle-imaging techniques for experimental fluid mechanics. Annu Rev Fluid Mech 23:261-304

Adrian RJ (2005) Twenty years of particle image velocimetry. Exp Fluids 39(2):159-169

Brown RG, Hwang PYC (1997) Introduction to random signals and applied Kalman filtering. Wiley, New York

Capart H, Young DL, Zech Y (2002) Voronoï imaging methods for the measurement of granular flows. Exp Fluids 32:121-135

Castellari S, Griffa A, Özgökmen TM, Poulain PM (2001) Prediction of particle trajectories in Adriatic Sea using Lagrangian data assimilation. J Mar Syst 29:33-50

Chiang TP, Sheu WH (1997) Numerical prediction of eddy structure in a shear-driven cavity. Comput Mech 20:379-396 
Chiang TP, Hwang RR, Sheu WH (1997) On end-wall corner vortices in a lid-driven cavity. J Fluids Eng 119:201-106

Chiang TP, Sheu WH, Hwang RR (1998) Effect of Reynolds number on the eddy structure in a lid-driven cavity. Int $\mathbf{J ~ N u}$ mer Methods Fluids 26:577-579

Douxchamps D, Devriendt D, Capart H, Craeye C, Macq B, Zech Y (2005) Stereoscopic and velocimetric reconstructions of the free surface topography of antidune flows. Exp Fluids 39(3):533-551

Guermond JL, Migeon C, Pineau G, Quartapelle L (2002) Start-up flows in a three-dimensional rectangular driven cavity of aspect ratio 1:1:2 at $\mathrm{Re}=1000$. J Fluid Mech 450:169-199

Guler M, Edil TB, Bosscher PJ (1999) Measurement of particle movement in granular soil using image analysis. J Comput Civ Eng 13:116-122

Han M, Kim C, Kim M, Lee S (1999) Particle migration in tube flow of suspensions. J Rheol 43(5):1157-1174

Högberg M, Chevalier M, Henningson DS (2003) Linear compensator control of a pointsource induced perturbation in a Falkner-Skan-Cooke boundary layer. Phys Fluids 15(8):24492452

Hu CC (2003) Analysis of motility for aquatic sperm in videomicroscopy. MSc Thesis, Department of Bio-Industrial Mechatronics Engineering, National Taiwan University

Ide K, Ghil M (1997) Extended Kalman filtering for vortex systems. Part I: methodology and point vortices. Dyn Atmos Oceans 27:301-332

Ito M, Tsujimichi S, Kosuge Y (2001) Tracking a three-dimensional moving target with distributed passive sensors using extended Kalman filter. Electron Commun Jpn Part 1 84(7):74-85

Jain R, Kasturi R, Schunck BG (1995) Machine vision. McGrawHill, New York

Kalman RE (1960) A new approach to linear filtering and prediction problems. Trans ASME J Basic Eng 82D:35-45

Kalman RE, Bucy RS (1961) New results in linear filtering and prediction problems. Trans ASME J Basic Eng 83D:95-108

Kieft RN, Schreel K, van der Plas G, Rindt C (2002) The application of a 3D PTV algorithm to a mixed convection flow. Exp Fluids 33:603-611

Koseff JR, Street RL (1984) The lid-driven flow: a synthesis of qualitative and quantitative observations. J Fluids Eng 106:390-398

Lauga E, Bewley TR (2004) Performance of a linear robust control strategy on a nonlinear model of spatially developing flows. J Fluid Mech 512:343-374
Liao JI (2002) Trajectory and velocity determination by smoother. MSc Thesis, Department of Electrical Engineering, National Taiwan University

Lo DC, Murugesan K, Young DL (2005) Numerical solutions of three-dimensional velocity-vorticity Navier-Stokes equations by finite difference method. Int $\mathbf{J}$ Numer Methods Fluids 47:1469-1487

Maas HG, Gruen A, Papantoniou D (1993) Particle tracking velocimetry in three-dimensional flows. Part 1: photogrammetric determination of particle coordinates. Exp Fluids 15:133-146

Matas J-P, Morris JF, Guazzelli E (2004) Inertial migration of rigid spherical particles in Poiseuille flow. J Fluid Mech 515:171-195

Migeon C (2002) Details on the start-up development of the Taylor-Gortler-like vortices inside a square-section lid-driven cavity for $1,000<\operatorname{Re}<3,200$. Exp Fluids 33:594-602

Murugesan K, Lo DC, Young DL (2005) An efficient global matrix free finite element algorithm for 3D flow problems. Commun Numer Methods Eng 21(3):107-118

Pan F, Acrivos A (1967) Steady flows in rectangular cavities. J Fluid Mech 28:643-655

Segré G, Silberberg A (1962) Behaviour of macroscopic rigid spheres in Poiseuille flow. Part 2. Experimental results and interpretation. J Fluid Mech 14:136-157

Shah M, Rangarajan K, Tsai PS (1993) Motion trajectories. IEEE Trans Syst Man Cybern 23(4):1138-1150

Shankar PN, Deshpande MD (2000) Fluid mechanics in the driven cavity. Annu Rev Fluid Mech 32:93-136

Sheu WH, Tsai SF (2002) Flow topology in a steady threedimensional lid-driven cavity. Comput Fluids 31:911-934

Spinewine B, Capart H, Larcher M, Zech Y (2003) Three-dimensional Voronoï imaging methods for the measurement of nearwall particulate flows. Exp Fluids 34:227-241

Ushijima S, Tanaka N (1996) Three-dimensional particle tracking velocimetry with laser-light sheet scannings. J Fluids Eng 118:352-357

Veber P, Dahl J, Hermansson R (1997) Study of the phenomena affecting the accuracy of a video-based particle tracking velocimetry technique. Exp Fluids 22:482-488

Virant M, Dracos T (1997) 3D PTV and its application on Lagrangian motion. Meas Sci Technol 8:1539-1552

Welch G, Bishop G (2001) An introduction to the Kalman filter. Course 8, SIGGRAPH 2001, Los Angeles, August 2001, ACM 